\title{
Performance of the KOTO Sampling Calorimeter
}

\author{
JunLee Kim for the KOTO Collaboration* \\ Chonbuk National Univeristy, Republic of Korea \\ E-mail: jikim1290@gmail.com
}

The purpose of the J-PARC KOTO experiment is to search for the $K_{L} \rightarrow \pi^{0} v \bar{v}$ decay which is sensitive to new physics. A main feature of the signal is two photons and nothing else observed in a hermetic detector system. Thus, it is important to detect all decay particles from the $K_{L}$ decays. A 5.5-m long cylindrical Lead/Scintillator sandwich calorimeter surrounds the $K_{L}$ decay region to detect extra photons. The detection efficiency of the sampling calorimeter was designed to meet the background elimination capability. We present the performance of the sampling calorimeter using tagged photons from the $K_{L} \rightarrow 3 \pi^{0}$ decay. In particular, the performance of a new sampling calorimeter installed in 2016 is reported.

ICHEP 2018, International Conference on High Energy Physics

4-11 July 2018

Seoul, Korea

${ }^{*}$ Speaker. 


\section{KOTO Experiment}

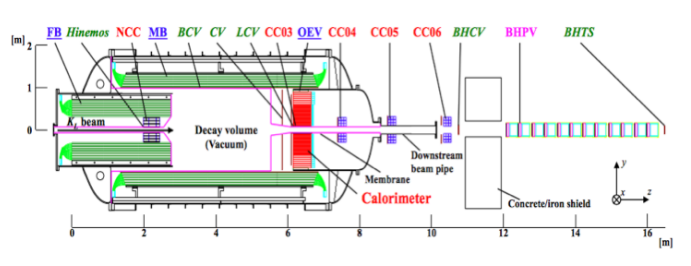

Figure 1: Cut-out-view of the KOTO detector in the physics run in 2015 .

The purpose of the KOTO experiment [1] is to search for the rare $K_{L}$ decay $K_{L} \rightarrow \pi^{0} v \bar{v}$ to explore new physics. The Standard Model predicts its branching ratio to be $(3.0 \pm 0.3) \times 10^{-11}$. Because this decay is calculated with extremely small theoretical uncertainty and highly suppressed in the Standard Model, the decay is a good probe to explore new physics. Observables from the $K_{L} \rightarrow \pi^{0} v \bar{v}$ decay are two photons and nothing else. The CsI calorimeter measures the energies and positions of the two photons, and hermetic veto counters ensure that no other particles exist.

\section{The Perforamce of The Photon Veto Counter}

A photon veto counter called Main Barrel (MB) surrounds the $K_{L}$ decay region to capture extra photons. It consists of layer of 1 or $2 \mathrm{~mm} \mathrm{~Pb}$ sheet and $5 \mathrm{~mm}$ scintillator, and its total thickness is $13.5 X_{0}$. An additional counter called the Inner Barrel (IB) was installed insde the MB to further suppress the background from the $K_{L} \rightarrow \pi^{0} \pi^{0}$ decay by a factor 3 with additional $5 X_{0}$

The performance of the barrel counters (MB and IB) was studied by using tagged photons. We used $K_{L} \rightarrow 3 \pi^{0}$ events with five photons in the CsI calorimeter to reconstruct the energy and direction of a photon going to the barrel. Using four photons detected in the calorimter, two $\pi^{0}$ 's were reconstructed to identify the decay vertex of $K_{L}$. With the momentum of the fifth photon in the calorimeter and kinematical constraints, the momentum of the sixth photon was reconstructed.

The hit timing of the sixth photon was calculated based on its estimated hit position and the timings of photons in the calorimeter. We first looked at the difference between the estimated and measured timings and aligned the measured timings as shown in Fig. 2 (a). We then evaluated the timing resolution of the barrel counters by comparing the estimated and measured timings as shown in Fig. 2 (b).

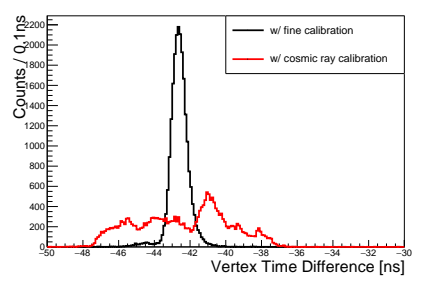

(a)

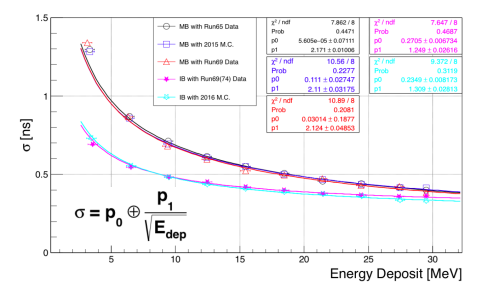

(b)

Figure 2: (a) Distribution of the difference between the estimated and measured timings of barrel counters. Results of implementation of timing calibration is presented in black distribution which is changed from red distribution. (b) Timing resolution of IB and MB. IB provides 1.5 times better timing resolution.

\section{References}

[1] J. K. Ahn et al., (J-PARC KOTO Collaboration), PTEP, 2017, 021 C01 (2017). 